Postprint version

Journal website

Pubmed link

DOI
https://www.sciencedirect.com/science/article/pii/S016885101830472X ?via\%3Dihub

: https://www.ncbi.nlm.nih.gov/pubmed/30224174

: $\quad$ 10.1016/j.healthpol.2018.09.006

This is a Nivel certified Post Print, more info at nivel.nl

\title{
A people-centred health workforce in Europe: How to make it happen?
}

\author{
Ellen Kuhlmann, Ronald Batenburg, Gilles Dussault
}

In 2015, a special issue of Health Policy addressed the question 'where are we going in health workforce governance in Europe?' [1]. The selected articles stressed the growing attention to the emergent health workforce research in Europe. The special issue presented complex and diverse health workforce reforms and concluded that there was a need for 'context-sensitive health workforce policies to creatively respond to "local" needs and efficiently use existing tools and expertise' [1, $p$. 1515]. In our summary we argued for integrated approaches, which moved 'the health workforce issues out of the shadow of interest strategies of professional and provider organizations' [1, p. 1516] and we highlighted the benefits of cross-country comparative research.

The present special issue now sets the focus on a people-centred health workforce in Europe and brings attention to issues of policy implementation, thus moving the debate one step further. This selection of research articles adds new knowledge and data that help deepen our understanding of how health system changes shape the development of the health workforce. Most progress can be observed in the emergence of new professional groups and in skills-mix changes. This special issue shows a better understanding of the complexity of factors that impact health workforce transformations, including new forms of stakeholder involvement and competence development beyond the traditional 'silo' approaches of the professions, a condition to move towards more integrated care and to building of a people-centred health workforce. However, challenges remain that constrain the creation of a people-centred health workforce.

The first section of the issue gives an overview of what matters in developing the future health workforce. The articles cover a broad range of countries, healthcare settings, and stakeholder groups, drawing from European comparative research. They bring into view supportive conditions for change and stakeholders involved. The research areas include primary care workforce development [2], interprofessional education and the relevance of culture [3], community orientation of general practitioners [4] and the relationships between new roles and patient views [5].

A second section reports on research into health workforce planning and skills-mix changes. Articles cover the inclusion of nurse practitioners and physician assistants in workforce planning in European countries [6], the reallocation of human resources in Portuguese public hospitals [7] and trends in work hour constraints in nursing in Germany [8]. The next section provides in-depth information on 
Kuhlmann, E., Batenburg, R., Dussault, G. A people-centred health workforce in Europe: How to make it happen? Health Policy: 2018, 122(10), 1053-1054

new professional groups and roles in Europe. The articles focus on healthcare assistants, a much under-researched group [9] and on the motivating and hindering factors for physicians, nurses and managers to undertake new roles in hospitals [10].

The final two sections discuss the challenges related to migration intentions of physicians and nurses in Lithuania [11] and to the relationship between dual practice, intention to work abroad and job satisfaction in Serbia [12]. Both cases highlight the potential threats that health workforce mobility and migration represent to less well-resourced healthcare systems in Europe. The final article directs our attention to the changing political landscapes and explores, in a comparative perspective, possible relationships between healthcare governance, professions and populism in EU countries [13].

This collection of articles provides new empirical evidence on policy levers and stakeholders, which may support the building of a people-centred workforce. The findings will help policymakers and researchers to understand better what works how and why, in developing a future health workforce that serves population needs, and to facilitate the implementation of new policies. The research also brings into view conditions which may have a negative impact or even counteract efforts to create a people-centred health workforce. One problem is that current health workforce policies in Europe still have no adequate response to mobility and migration flows of healthcare workers. Another serious issue is growing right-wing populism, which needs greater attention to identify and effectively prevent threats to public health and the health workforce. It is time to recall that the health workforce must be placed on top of the agenda of health policymaking both globally and locally [1].

This special issue is closely connected to the work of the European Public Health Association (EUPHA) section on 'Health Workforce Research'. We wish to thank the Editor-in-Chief, Reinhard Busse, for increasing the visibility of health workforce research and Julia Röttger and the Health Policy team at TU Berlin and Elsevier for their support in putting this special issue together. We also thank authors for their collaboration and the anonymous reviewers for contributing their time and helpful comments.

\section{References}

[1] E. Kuhlmann, R. Batenburg, G. Dussault, Guest Editorial Health workforce governance in Europe: where are we going? Health Policy, 119 (12) (2015), pp. 1515-1516

[2] E. Kuhlmann, P.P. Groenewegen, C. Bond, V. Burau, D.J. Hunter Primary care workforce development in Europe: an overview of health system responses and stakeholder views Health Policy, 122 (10) (2018), pp. 1055-1062

[3] M. Bonello, J. Morris, N. Azzopardi Muscat The role of national culture in shaping health workforce collaboration: Lessons learned from a case study on attitudes to interprofessional education in Malta Health Policy, 122 (10) (2018), pp. 1063-1069

[4] L. Vermeulen, W. Schäfer, D.R. Pavlic, P. Groenewegen Community orientation of general practitioners in 34 countries Health Policy, 122 (10) (2018), pp. 1070-1077

[5] M. Ruggeri, C. Drago, V. Moramarco, S. Coretti, J. Köppen, K. Islam, et al. New professional roles and patient satisfaction: evidence from a European survey along three clinical pathways Health Policy, 122 (10) (2018), pp. 1078-1084

[6] C.B. Maier, R. Batenburg, S. Birch, B. Zander, R. Elliott, R. Busse, et al. Health workforce planning: which countries include Nurse Practitioners and Physician Assistants and with which effects? Health Policy, 122 (10) (2018), pp. 1085-1092

[7] D. Ferreira, A.M. Nunes, R.C. Marques Doctors, nurses, and the optimal scale size in the Portuguese public hospitals Health Policy, 122 (10) (2018), pp. 1093-1100 
Kuhlmann, E., Batenburg, R., Dussault, G. A people-centred health workforce in Europe: How to make it happen? Health Policy: 2018, 122(10), 1053-1054

[8] M. Alameddine, S. Otterbach, B. Rafii, A. Sousa-Poza Work hour constraints in the German nursing workforce: A quarter of a century in review Health Policy, 122 (10) (2018), pp. 1101-1108 [9] M. Kroezen, W. Schäfer, W. Sermeus, J. Hansen, R. Batenburg Healthcare assistants in EU Member States: an overview Health Policy, 122 (10) (2018), pp. 1109-1117

[10] J. Koeppen, C.B. Maier, R. Busse, MUNROS team, C. Bond, MUNROS CO-PI, et al. What are the motivating and hindering factors for health professionals to undertake new roles in hospitals? A study among physicians, nurses and managers looking at breast cancer and acute myocardial infarction care in nine countries Health Policy, 122 (10) (2018), pp. 1118-1125

[11] B. Goštautaitè, I. Bučiūnienè, Z. Milašauskienè, K. Bareikis, E. Bertašiūtè, G. Mikelionienè Migration intentions of Lithuanian physicians, nurses, residents and medical students Health Policy, 122 (10) (2018), pp. 1126-1131

[12] M. Gacevic, M. Santric Milicevic, M. Vasic, V. Horozovic, M. Milicevic, N. Milic The relationship between dual practice, intention to work abroad, and job satisfaction: a population-based study in the Serbian public healthcare sector Health Policy, 122 (10) (2018), pp. 1132-1139

[13] E. Pavolini, E. Kuhlmann, T.I. Agartan, V. Burau, R. Mannion, E. Speed Healthcare governance, professions and populism: is there a relationship? An explorative comparison of five European countries Health Policy, 122 (10) (2018), pp. 1140-1148 